\title{
Time variation of the fine structure constant and of the Higgs vacuum expectation value on cosmological time scales
}

\author{
M. E. Mosquera ${ }^{1,2}$ and O. Civitarese ${ }^{2}$ \\ ${ }^{1}$ Facultad de Ciencias Astronómicas y Geofísicas, Universidad Nacional de La Plata, Paseo del Bosque, (1900) La Plata, Argentina \\ e-mail: osvaldo.civitarese@fisica.unlp.edu.ar \\ 2 Department of Physics, University of La Plata c.c. 67 (1900), La Plata, Argentina
}

Received 16 November 2010 / Accepted 29 November 2010

\begin{abstract}
Aims. We study the time variation of both the fine structure constant, $\alpha$, and the Higgs vacuum expectation value, $v$, during Big Bang nucleosynthesis $(\mathrm{BBN})$.

Methods. We calculate the primordial abundances of $\mathrm{D},{ }^{4} \mathrm{He}$, and ${ }^{7} \mathrm{Li}$ by modifying Kawano's code, and by using observational data we set constraints on the joint variation of $\alpha$ and $v$. To perform the calculations we considered the dependence of the deuteriumbinding energy, $\epsilon_{\mathrm{D}}$, upon $v$, obtained from the treatment of different proton-neutron interactions.

Results. Results are consistent with variation on $v$ (even at the level of $6 \sigma$ ) and null variation of $\alpha$ (within $2 \sigma$ ) if the ${ }^{7} \mathrm{Li}$ data are used in the analysis. However, if data on these nuclei are not considered in the statistical analysis, we found null variation on both fundamental constants within $2 \sigma$.

Conclusions. We found that the best-fit values of the variation of $v$ and $\alpha$ are sensible to the dependence of the deuterium binding energy upon the Higgs vacuum expectation value. We found non-null variation of $v$ within $6 \sigma$ if all the observational data are used in the analysis. If data on the primordial abundance of ${ }^{7} \mathrm{Li}$ are taken at face value, the discrepancy between BBN and WMAP estimates may be explained by allowing variations of $v$.
\end{abstract}

Key words. primordial nucleosynthesis - nuclear reactions - nucleosynthesis - abundances - cosmology: theory

\section{Introduction}

Several observations can establish limits to the variation of different fundamental constants, such as the atomic clocks (Bize et al. 2003; Fischer et al. 2004; Peik et al. 2004; Prestage et al. 1995; Sortais et al. 2000; Marion et al. 2003), the Oklo natural fission reactor (Damour \& Dyson 1996; Fujii et al. 2000) and the analysis of the spectra of quasar absorption systems. These astronomical observations suggest a possible variation of the fine structure constant and the electron-to-proton mass ratio (Webb et al. 1999, 2001; Murphy et al. 2001a,b, 2003; Ivanchik et al. 2005; Reinhold et al. 2006; Tzanavaris et al. 2007). However, another analysis of similar astronomical data gives null variation of the fine structure constant (Martínez Fiorenzano et al. 2003; Quast et al. 2004; Bahcall et al. 2004; Srianand et al. 2004; King et al. 2008; Thompson et al. 2009; Malec et al. 2010).

The Big Bang nucleosynthesis (BBN) is a useful tool to study the time variation of fundamental constants, such as the fine structure constant, $\alpha$, the Higgs vacuum expectation value, $v$, and the Planck mass, among others. Several theories that attempt to unify the four fundamental interactions, such as superstrings (Wu \& Wang 1986; Barr \& Mohapatra 1988; Maeda 1988; Damour \& Polyakov 1994; Damour et al. 2002a,b), brane world (Youm 2001a,b; Brax et al. 2003; Palma et al. 2003) and Kaluza-Klein theories (Kaluza 1921; Klein 1926; Weinberg 1983; Gleiser \& Taylor 1985; Overduin \& Wesson 1997), allow fundamental constants to vary within cosmological time scales. The time variation of several fundamental constants was studied by Campbell \& Olive (1995), Bergström et al. (1999), Ichikawa \& Kawasaki (2002), Nollett \& Lopez (2002), Yoo \& Scherrer (2003), Müller et al. (2004), Ichikawa \& Kawasaki (2004), Cyburt et al. (2005), Landau et al. (2006), Coc et al. (2007), Chamoun et al. (2007), Mosquera et al. (2008), Landau et al. (2008), Landau \& Scóccola (2010), Mosquera \& Civitarese (2010), Civitarese et al. (2010), among others.

If the Higgs vacuum expectation value acquires a different value during $\mathrm{BBN}$ than the present one, the electron mass, the proton-neutron mass difference, the Fermi constant, and the deuterium binding energy, $\epsilon_{\mathrm{D}}$, will be different from the corresponding actual values. Several authors have studied the dependence of $\epsilon_{\mathrm{D}}$ on $v$ (Flambaum \& Shuryak 2002, 2003; Dmitriev \& Flambaum 2003; Beane \& Savage 2003; Epelbaum et al. 2003; Yoo \& Scherrer 2003; Dmitriev et al. 2004; Flambaum \& Wiringa 2007; Berengut et al. 2010; Mosquera \& Civitarese 2010; Civitarese et al. 2010).

In this work, we study the effects of a possible variation of the fine structure constant and of the Higgs vacuum expectation value, considering a fixed value of the strong-coupling constant $\Lambda_{\mathrm{QCD}}$. To perform the calculation of the primordial abundances, we use the linear dependence of $\epsilon_{\mathrm{D}}$ with $v$ discussed in Mosquera \& Civitarese (2010); Civitarese et al. (2010). We use observational data of $\mathrm{D},{ }^{4} \mathrm{He}$, and ${ }^{7} \mathrm{Li}$ to obtain constraints on the variation of the participant fundamental constants. We also perform an analysis of the sensibility of these constraints on the lithium abundance and the dependencies between $\epsilon_{\mathrm{D}}$ and $v$. 
Table 1. Values of the coefficient $\kappa$ of Eq. (1).

\begin{tabular}{lc}
\hline \hline Potential & $\kappa$ \\
\hline Argonne & -1.23 \\
Bonn & -0.66 \\
Nijmegen & -1.66 \\
Reid & -1.83 \\
\hline
\end{tabular}

This work is organized as follows. In Sect. 2 we calculate the primordial abundances and obtain constraints on the joint variation of $\alpha$ and $v$. The conclusions are presented in Sect. 3 .

\section{Bounds from BBN}

In this section we study the effect of a possible variation of the fine structure constant, $\alpha$, and of the Higgs vacuum expectation value, $v$, during primordial nucleosynthesis. If $\alpha$ acquires a value different from the actual one, the neutron-to-proton ratio, the neutron-proton mass difference, the weak decay rates, and the cross sections of several reactions involved during BBN would be different from current estimates (Landau et al. 2008), and consequently, the primordial abundances would be modified. The electron mass, the Fermi constant, the neutron-proton mass difference, the weak decay rates, the masses of light nuclei, the deuterium binding energy, $\epsilon_{\mathrm{D}}$, and the initial abundance of deuterium will be modified if the Higgs vacuum expectation value varies with time (Landau et al. 2008; Mosquera \& Civitarese 2010).

The dependence of the deuterium-binding energy on the Higgs vacuum expectation value is model dependent (Flambaum \& Wiringa 2007). In this work, we use the results obtained by Mosquera \& Civitarese (2010); Civitarese et al. (2010). The authors had calculated the constant of proportionality, $\kappa$, which relates $\epsilon_{\mathrm{D}}$ and $v$, for four different nucleon-nucleon potentials (Argonne $v_{18}$ potential, Bonn potential, Nijmegen potential and Reid 93 potential),

$\frac{\Delta \epsilon_{\mathrm{D}}}{\left(\epsilon_{\mathrm{D}}\right)_{0}}=\kappa \frac{\Delta v}{v_{0}}$

where $\Delta \epsilon_{\mathrm{D}}=\left(\epsilon_{\mathrm{D}}\right)_{\mathrm{BBN}}-\left(\epsilon_{\mathrm{D}}\right)_{0}$, and $\Delta v=v_{\mathrm{BBN}}-v_{0}$. The subindexes $\mathrm{BBN}$ and 0 indicate the value of the constant at primordial nucleosynthesis and at the present time, respectively. In Table 1 we present the values of $\kappa$ obtained by Mosquera \& Civitarese (2010); Civitarese et al. (2010). The Argonne $v_{18}$ potential includes an electromagnetic interaction (Wiringa et al. 1995), proportional to the fine structure constant. We modified this potential to include the variation of the fine structure constant and performed the calculation of $\epsilon_{\mathrm{D}}$. We obtained the relation $\frac{\Delta \epsilon_{\mathrm{D}}}{\left(\epsilon_{\mathrm{D}}\right)_{0}}=-0.0019 \frac{\Delta \alpha}{\alpha_{0}}$.

In order to calculate the primordial abundances, we modified the code developed by Kawano (Kawano 1988, 1992) for each nucleon-nucleon potential of Table 1 (Mosquera \& Civitarese 2010; Civitarese et al. 2010). For details about the corrections introduced in the public version of Kawano's code see Mosquera et al. (2008); Landau et al. (2008); Mosquera \& Civitarese (2010).

The WMAP data are able to constraint the baryon density $\Omega_{\mathrm{B}} h^{2}$ (related to the baryon-to-photon ratio $\eta_{\mathrm{B}}$ ) with great accuracy, but there is still some degeneracy between the model parameters, namely: one is dealing with a parametric hypersurface defined by the values of $\Omega_{\mathrm{B}} h^{2}, \Omega_{\mathrm{CDM}} h^{2}$ (dark matter density in units of the critical density), $\Theta$ (gives the ratio of the comoving
Table 2. Best-fit parameter values for the BBN constraints.

\begin{tabular}{cccc}
\hline \hline$\kappa$ & $\eta_{\mathrm{B}} \pm \sigma\left[10^{-10}\right]$ & $\frac{\Delta \alpha}{\alpha_{0}} \pm \sigma\left[10^{-3}\right]$ & $\frac{\Delta v}{v_{0}} \pm \sigma\left[10^{-3}\right]$ \\
\hline-1.23 & $6.440_{-0.219}^{+0.382}$ & $-2.5 \pm 4.8$ & $29.5_{-1.1}^{+1.3}$ \\
-0.66 & $6.150_{-0.209}^{+0.365}$ & $-8.5_{-4.7}^{+4.5}$ & $29.9_{-1.1}^{+1.3}$ \\
-1.66 & $6.744_{-0.378}^{+0.317}$ & $1.5_{-4.0}^{+4.7}$ & $29.6_{-1.3}^{+1.1}$ \\
-1.83 & $6.901_{-0.386}^{+0.243}$ & $3.0 \pm 4.0$ & $29.6_{-1.4}^{+1.0}$ \\
\hline
\end{tabular}

sound horizon at decoupling to the angular diameter distance to the surface of last scattering), $\tau$ (reionization optical depth), $n_{\mathrm{s}}$ (scalar spectral index), $A_{\mathrm{s}}$ (amplitude of the density fluctuations). For this reason we computed the light nuclei abundances for the following cases:

i) variation of $\alpha$ and $v$ allowing $\eta_{\mathrm{B}}$ vary,

ii) variation of $\alpha$ and $v$ keeping $\eta_{\mathrm{B}}$ fixed at WMAP value $\left(\eta_{\mathrm{B}}^{\mathrm{WMAP}}=(6.108 \pm 0.219) \times 10^{-10}\right) \quad($ Spergel et al. 2003, 2007).

In order to obtain the best-fit values for the parameters, we performed a $\chi^{2}$-test to compare the theoretical abundances and the observational data.

The observational data for D were extracted from Burles \& Tytler (1998a,b), O’Meara et al. (2001), Pettini \& Bowen (2001), Levshakov et al. (2002), Kirkman et al. (2003), Crighton et al. (2004), O’Meara et al. (2006), Pettini et al. (2008), Ivanchik et al. (2010).

We used the data from Izotov et al. (2006), Peimbert et al. (2007), Izotov et al. (2007), Izotov \& Thuan (2010) for ${ }^{4} \mathrm{He}$, and for ${ }^{7} \mathrm{Li}$ we considered the data given by Bonifacio \& Molaro (1997), Molaro et al. (1997), Ryan et al. (2000), Bonifacio et al. (2002), Boesgaard et al. (2005), Asplund et al. (2006), Bonifacio et al. (2007), Hosford et al. (2009). Regarding the consistency of the data, we followed the treatment of Yao et al. (2006) and increased the errors by a fixed factor: $\Theta_{\mathrm{D}}=2.37, \Theta^{4} \mathrm{He}=2.69$ and $\Theta_{7} \mathrm{Li}=1.43$ for $\mathrm{D},{ }^{4} \mathrm{He}$ and ${ }^{7} \mathrm{Li}$, respectively.

\subsection{Variation of $\alpha$ and $v$ allowing $\eta_{B}$ to vary}

We calculated the BBN abundances for different values of the fine structure constant, the Higgs vacuum expectation value and $\eta_{\mathrm{B}}$, for each potential considered (see Table 1). We performed a $\chi^{2}$-test to find the best-fit values of the parameters. In Table 2 we show the results of the parameters corresponding to the best fit, at $1 \sigma$, for the BBN constraints on $\eta_{\mathrm{B}}$ (in units of $10^{-10}$ ), $\frac{\Delta \alpha}{\alpha_{0}}$ (in units of $10^{-3}$ ) and $\frac{\Delta v}{v_{0}}$ (in units of $10^{-3}$ ). The values were obtained for the different values of $\kappa$ given in Table 1. For all these cases, the value of $\frac{\chi_{\min }^{2}}{N-3}$ is 1.00 .

We found good agreement at the level of three standard deviations between our best-fit value of $\eta_{\mathrm{B}}$ and the one obtained using WMAP data (Spergel et al. 2007). We also found null variation of $\alpha$ within two standard deviations, and variation of the Higgs vacuum expectation value, even at the level of six standard deviations. In Fig. 1 we present the $3 \sigma$-likelihood contours for $\eta_{\mathrm{B}}, \frac{\Delta \alpha}{\alpha_{0}}$ and $\frac{\Delta v}{v_{0}}$, and the one-dimensional likelihood for all potentials.

\subsection{Variation of $\alpha$ and $v$ keeping $\eta_{B}$ fixed}

Once again, we calculated the BBN abundances for different values of the fine structure constant and of the Higgs vacuum 
M. E. Mosquera and O. Civitarese: Time variation of $\alpha$ and $v$ on cosmological time scales

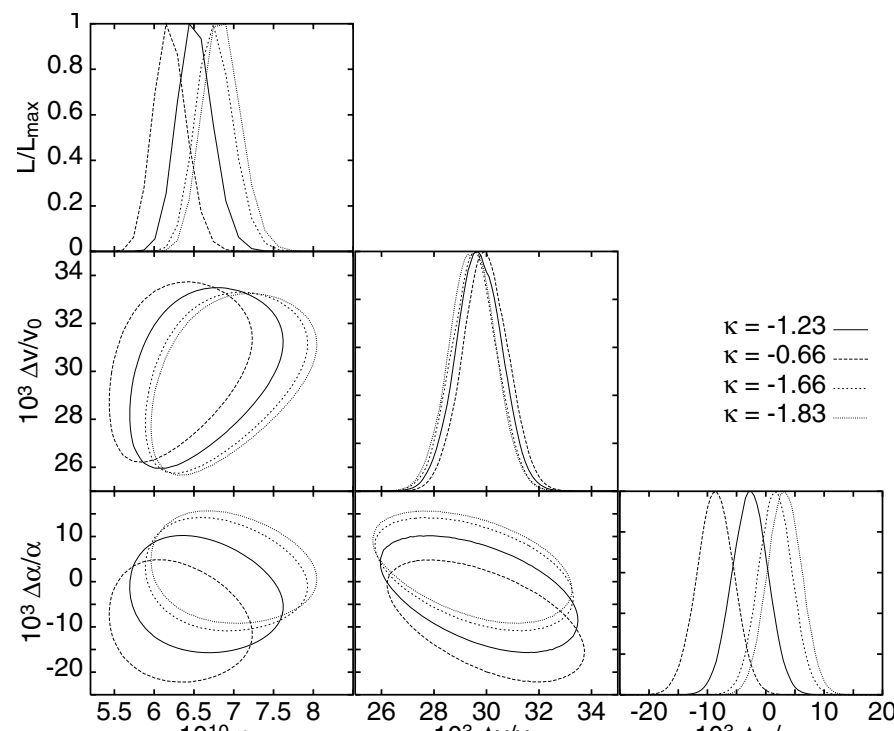

Fig. 1. $3 \sigma$ Likelihood contours for $\frac{\Delta \alpha}{\alpha_{0}}, \frac{\Delta v}{v_{0}}$ and $\eta_{\mathrm{B}}$ and one-dimensional likelihood for all values of $\kappa$.

Table 3. Best-fit parameters with $\eta_{\mathrm{B}}$ fixed by WMAP.

\begin{tabular}{cccc}
\hline \hline$\kappa$ & $\frac{\Delta \alpha}{\alpha_{0}} \pm \sigma\left[10^{-3}\right]$ & $\frac{\Delta v}{v_{0}} \pm \sigma\left[10^{-3}\right]$ & $\frac{\chi_{\min }^{2}}{N-2}$ \\
\hline-1.23 & $-2.0 \pm 4.0$ & $29.0 \pm 1.5$ & 1.04 \\
-0.66 & $-9.0 \pm 5.0$ & $29.5 \pm 2.0$ & 0.96 \\
-1.66 & $3.0 \pm 4.0$ & $28.5 \pm 1.5$ & 1.19 \\
-1.83 & $5.0 \pm 4.0$ & $28.0 \pm 2.0$ & 1.25 \\
\hline
\end{tabular}

expectation value for each potential considered (see Table 1). This calculation was made keeping $\eta_{\mathrm{B}}$ fixed at WMAP value $\left(\eta_{\mathrm{B}}^{\text {WMAP }}=(6.108 \pm 0.219) \times 10^{-10}\right)($ Spergel et al. 2007). We performed a $\chi^{2}$-test to find the best-fit value. In Table 3 we present the results for the best-fit parameter values within one standard deviation for the BBN constraints on $\frac{\Delta \alpha}{\alpha_{0}}$ (in units of $10^{-3}$ ) and $\frac{\Delta v}{v_{0}}$ (in units of $10^{-3}$ ), with $\eta_{\mathrm{B}}$ fixed at the WMAP estimate, for the different values of $\kappa$, as given in Table 1 .

In Fig. 2 we present the $3 \sigma$-likelihood contours for $\frac{\Delta \alpha}{\alpha_{0}}$ and $\frac{\Delta v}{v_{0}}$ and one-dimensional likelihood, for all potentials.

We found null variation of $\alpha$ at $2 \sigma$ level, but the variation of $v$ is non-null even at $6 \sigma$. If the analysis does not include the lithium-data-set, we found null variation of both fundamental constants at the level of $2 \sigma$.

\section{Conclusion}

We obtained bounds on the joint variation of $\alpha$ and $v$ using the observational abundances of $\mathrm{D},{ }^{4} \mathrm{He}$, and ${ }^{7} \mathrm{Li}$. We performed the analysis for different estimates of the dependence of the deuterium-binding energy on the Higgs vacuum expectation value. We found that the four dependencies considered, that is for the four values of $\kappa$ of Table 1, give similar results. We found reasonable fits for the variation of $\alpha, v$ and $\eta_{\mathrm{B}}$ for the whole data set. However, and referring to the particular case of $v$, its variation is significant only when the data-set includes the ${ }^{7} \mathrm{Li}$ abundance. If the present values of ${ }^{7} \mathrm{Li}$ abundances are correct (Meléndez \& Ramírez 2004; Richard et al. 2005; Prodanović \& Fields 2007), then varying fundamental constants

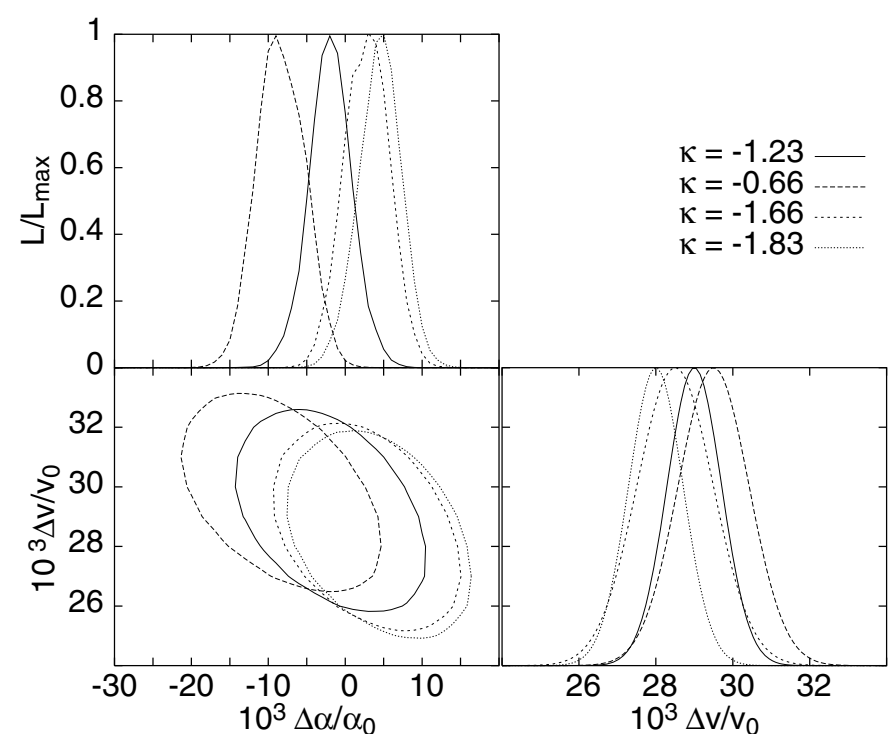

Fig. 2. $3 \sigma$ Likelihood contours for $\frac{\Delta \alpha}{\alpha_{0}}$ vs. $\frac{\Delta v}{v_{0}}$ and one-dimensional likelihood for all values of $\kappa$.

would be a possible explanation to solve the discrepancy between the light element abundances and the WMAP estimates.

Acknowledgements. Support for this work was provided by the PIP 0740 of the National Research Council (CONICET) of Argentina. The authors are members of the Scientific Research Career of the CONICET.

\section{References}

Asplund, M., Lambert, D. L., Nissen, P. E., Primas, F., \& Smith, V. V. 2006, ApJ, 644, 229

Bahcall, J. N., Steinhardt, C. L., \& Schlegel, D. 2004, ApJ, 600, 520

Barr, S. M., \& Mohapatra, P. K. 1988, Phys. Rev. D, 38, 3011

Beane, S. R., \& Savage, M. J. 2003, Nucl. Phys. A, 713, 148

Berengut, J. C., Flambaum, V. V., \& Dmitriev, V. F. 2010, Phys. Lett. B, 683, 114

Bergström, L., Iguri, S., \& Rubinstein, H. 1999, Phys. Rev. D, 60, 45005

Bize, S., Diddams, S. A., Tanaka, U., et al. 2003, Phys. Rev. Lett., 90, 150802

Boesgaard, A. M., Novicki, M. C., \& Stephens, A. 2005, in From Lithium to Uranium: Elemental Tracers of Early Cosmic Evolution, ed. V. Hill, P. François, \& F. Primas, IAU Symp., 228, 29

Bonifacio, P., \& Molaro, P. 1997, MNRAS, 285, 847

Bonifacio, P., Pasquini, L., Spite, F., et al. 2002, ApJ, 390, 91

Bonifacio, P., Molaro, P., Sivarani, T., et al. 2007, A\&A, 462, 851

Brax, P., van de Bruck, C., Davis, A.-C., \& Rhodes, C. S. 2003, Astro. Nucl. Space Sci., 283, 627

Burles, S., \& Tytler, D. 1998a, ApJ, 499, 699

Burles, S., \& Tytler, D. 1998b, ApJ, 507, 732

Campbell, B. A., \& Olive, K. A. 1995, Phys. Lett. B, 345, 429

Chamoun, N., Landau, S. J., Mosquera, M. E., \& Vucetich, H. 2007, J. Phys. G Nucl. Phys., 34, 163

Civitarese, O., Moliné, M. A., \& Mosquera, M. E. 2010, Nucl. Phys., A, 846, 157

Coc, A., Nunes, N. J., Olive, K. A., Uzan, J.-P., \& Vangioni, E. 2007, Phys. Rev. D, 76, 023511

Crighton, N. H. M., Webb, J. K., Ortiz-Gil, A., \& Fernández-Soto, A. 2004, MNRAS, 355, 1042

Cyburt, R. H., Fields, B. D., Olive, K. A., \& Skillman, E. 2005, Astropart. Phys., 23,313

Damour, T., \& Dyson, F. 1996, Nucl. Phys. B, 480, 37

Damour, T., \& Polyakov, A. M. 1994, Nucl. Phys. B, 95, 10347

Damour, T., Piazza, F., \& Veneziano, G. 2002a, Phys. Rev. Lett., 89, 081601

Damour, T., Piazza, F., \& Veneziano, G. 2002b, Phys. Rev. D, 66, 046007

Dmitriev, V. F., \& Flambaum, V. V. 2003, Phys. Rev. D, 67, 063513

Dmitriev, V. F., Flambaum, V. V., \& Webb, J. K. 2004, Phys. Rev. D, 69, 063506

Epelbaum, E., Meißner, U., \& Glöckle, W. 2003, Nucl. Phys. A, 714, 535

Fischer, M., Kolachevsky, N., Zimmermann, M., et al. 2004, Phys. Rev. Lett., 92, 230802 
Flambaum, V. V., \& Shuryak, E. V. 2002, Phys. Rev. D, 65, 103503 Flambaum, V. V., \& Shuryak, E. V. 2003, Phys. Rev. D, 67, 083507 Flambaum, V. V., \& Wiringa, R. B. 2007, Phys. Rev. C, 76, 054002 Fujii, Y., Iwamoto, A., Fukahori, T., et al. 2000, Nucl. Phys. B, 573, 377 Gleiser, M., \& Taylor, J. G. 1985, Phys. Rev. D, 31, 1904

Hosford, A., Ryan, S. G., García Pérez, A. E., Norris, J. E., \& Olive, K. A. 2009, A\&A, 493, 601

Ichikawa, K., \& Kawasaki, M. 2002, Phys. Rev., D65, 123511

Ichikawa, K., \& Kawasaki, M. 2004, Phys. Rev. D, 69, 123506

Ivanchik, A., Petitjean, P., Varshalovich, D., et al. 2005, A\&A, 440, 45

Ivanchik, A. V., Petitjean, P., Balashev, S. A., et al. 2010, MNRAS, Soc., 297

Izotov, Y. I., \& Thuan, T. X. 2010, ApJ, 710, L67

Izotov, Y. I., Schaerer, D., Blecha, A., et al. 2006, A\&A, 459, 71

Izotov, Y. I., Thuan, T. X., \& Stasińska, G. 2007, ApJ, 662, 15

Kaluza, T. 1921, Sitzungber. Preuss. Akad. Wiss. K, 1, 966

Kawano, L. 1988, fERMILAB-PUB-88-034-A

Kawano, L. 1992, fERMILAB-PUB-92-004-A

King, J. A., Webb, J. K., Murphy, M. T., \& Carswell, R. F. 2008, Phys. Rev. Lett., 101, 251304

Kirkman, D., Tytler, D., Suzuki, N., O’Meara, J. M., \& Lubin, D. 2003, ApJSS, 149,1

Klein, O. 1926, Z. Phys., 37, 895

Landau, S. J., \& Scóccola, G. 2010, A\&A, 517, A62

Landau, S. J., Mosquera, M. E., \& Vucetich, H. 2006, ApJ, 637, 38

Landau, S. J., Mosquera, M. E., Scóccola, C. G., \& Vucetich, H. 2008, Phys. Rev. D, 78, 083527

Levshakov, S. A., Dessauges-Zavadsky, M., D’Odorico, S., \& Molaro, P. 2002, ApJ, 565, 696

Müller, C. M., Schäfer, G., \& Wetterich, C. 2004, Phys. Rev. D, 70, 083504

Maeda, K. 1988, Modern Phys. Lett. A, 31, 243

Malec, A. L., Buning, R., Murphy, M. T., et al. 2010, MNRAS, 403, 1541

Marion, H., Pereira Dos Santos, F., Abgrall, M., et al. 2003, Phys. Rev. Lett., 90, 150801

Martínez Fiorenzano, A. F., Vladilo, G., \& Bonifacio, P. 2003, Soc. Astron. Ital. Mem. Suppl., 3, 252

Meléndez, J., \& Ramírez, I. 2004, ApJ, 615, L33

Molaro, P., Bonifacio, P., \& Pasquini, L. 1997, MNRAS, 292, L1

Mosquera, M. E., \& Civitarese, O. 2010, A\&A, 520, A112

Mosquera, M. E., Scóccola, C., Landau, S., \& Vucetich, H. 2008, A\&A, 478, 675

Murphy, M. T., Webb, J. K., Flambaum, V. V., et al. 2001a, MNRAS, 327, 1208
Murphy, M. T., Webb, J. K., Flambaum, V. V., Prochaska, J. X., \& Wolfe, A. M. 2001b, MNRAS, 327, 1237

Murphy, M. T., Webb, J. K., \& Flambaum, V. V. 2003, MNRAS, 345, 609

Nollett, K. M., \& Lopez, R. E. 2002, Phys. Rev., D66, 063507

O'Meara, J. M., Tytler, D., Kirkman, D., et al. 2001, ApJ, 552, 718

O’Meara, J. M., Burles, S., Prochaska, J. X., et al. 2006, ApJ, 649, L61

Overduin, J. M., \& Wesson, P. S. 1997, Phys. Rep., 283, 303

Palma, G. A., Brax, P., Davis, A. C., \& van de Bruck, C. 2003, Phys. Rev. D, 68 123519

Peik, E., Lipphardt, B., Schnatz, H., et al. 2004, Phys. Rev. Lett., 93, 170801

Peimbert, M., Luridiana, V., \& Peimbert, A. 2007, ApJ, 666, 636

Pettini, M., \& Bowen, D. V. 2001, ApJ, 560, 41

Pettini, M., Zych, B. J., Murphy, M. T., Lewis, A., \& Steidel, C. C. 2008, MNRAS, 391, 1499

Prestage, J. D., Tjoelker, R. L., \& Maleki, L. 1995, Phys. Rev. Lett., 74, 3511

Prodanović, T., \& Fields, B. D. 2007, Phys. Rev. D, 76, 083003

Quast, R., Reimers, D., \& Levshakov, S. A. 2004, A\&A, 415, L7

Reinhold, E., Buning, R., Hollenstein, U., et al. 2006, Phys. Rev. Lett., 96, 151101

Richard, O., Michaud, G., \& Richer, J. 2005, ApJ, 619, 538

Ryan, S. G., Beers, T. C., Olive, K. A., Fields, B. D., \& Norris, J. E. 2000, ApJ, 530, L57

Sortais, Y., Bize, S., Abgrall, M., et al. 2000, Phys. Scripta, T95, 50 Spergel, D. N., Verde, L., Peiris, H. V., et al. 2003, ApJSS, 148, 175 Spergel, D. N., Bean, R., Doré, O., et al. 2007, ApJSS, 170, 377

Srianand, R., Chand, H., Petitjean, P., \& Aracil, B. 2004, Phys. Rev. Lett., 92, 121302

Thompson, R. I., Bechtold, J., Black, J. H., et al. 2009, ApJ, 703, 1648

Tzanavaris, P., Murphy, M. T., Webb, J. K., Flambaum, V. V., \& Curran, S. J. 2007, MNRAS, 374, 634

Webb, J. K., Flambaum, V. V., Churchill, C. W., Drinkwater, M. J., \& Barrow, J. D. 1999, Phys. Rev. Lett., 82, 884

Webb, J. K., Murphy, M. T., Flambaum, V. V., et al. 2001, Phys. Rev. Lett., 87, 091301

Weinberg, S. 1983, Phys. Lett. B, 125, 265

Wiringa, R. B., Stoks, V. G. J., \& Schiavilla, R. 1995, Phys. Rev. C, 51, 38

Wu, Y., \& Wang, Z. 1986, Phys. Rev. Lett., 57, 1978

Yao, W.-M., Amsler, C., Asner, D., et al. 2006, J. Phys. G, 33, 1

Yoo, J. J., \& Scherrer, R. J. 2003, Phys. Rev. D, 67, 043517

Youm, D. 2001a, Phys. Rev. D, 63, 125011

Youm, D. 2001b, Phys. Rev. D, 64, 085011 